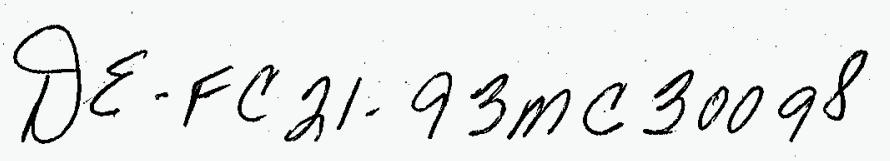

Final Report: August 1998

\title{
Characterizing Soil/Water Sorption and Desorption Behavior of BTEX and PAHs using Selective Supercritical Fluid Extraction (SFE)
}

Submitted to: Harley Hopkins, American Petroleum Institute John Harju, Gas Research Institute Paula Flenory, Federal Energy Technology Center

$\begin{array}{ll}\text { Submitted by: } & \begin{array}{l}\text { Steve Hawthorne, Energy and Environmental Research Center } \\ \text { University of North Dakota, Grand Forks, ND 58201-9018 } \\ \text { Phone: 701-777-5256, Fax: 701-777-5181 }\end{array} \\ \text { I. Summary: } & \end{array}$

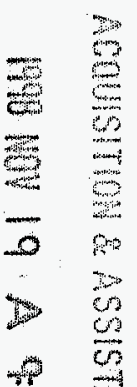

The first goal of the proposed study was to generate initial data to determine the ability selective SFE behavior to mimic the soil/water sorption and desorption behavior of BTEX (benzene, toluene, and xylenes) and PAHs (polycyclic aromatic hydrocarbons). Samples generated by Professor Bill Rixey's column sorption studies (aged for 2 weeks to 8 months) and desorption studies (six weeks desorption of the aged soil columns with pure water) were extracted using sequentially-stronger SFE conditions to selectively remove different fractions of each BTEX and PAH component which range from "loosely" to "tightly" bound in the soil matrices. The selective SFE results parallel the sorption/desorption leaching behavior and mechanisms determined by Professor Rixey's investigations (under separate funding) using water desorption of soil columns previously aged with BTEX and PAHs. These results justify more intensive investigations of the use of selective SFE to mimic soil/water sorption and desorption of organic pollutants related to fossil fuels which will be performed under separate funding.

The second goal of the study was to determine if selective SFE extraction behavior parallels the remediation behavior displayed by PAHs currently undergoing in-situ bioremediation at a manufactured gas plant (MGP) site. Based on soil analyses of several individual PAHs (as well as total PAHs) before remediation began, and after 147 days of remediation, selective SFE successfully mimicked remediation behavior. These results strongly support the use of selective SFE to predict remediation behavior of soils contaminated with PAHs, and are expected to provide a powerful and rapid analytical tool which will be useful for determining the remediation endpoints which are necessary for environmental protection. Based on the initial success found in the present study, additional investigations into the use of SFE for predicting and monitoring the remediation behavior of PAHcontaminated soils will be performed under separate funding.

\section{Approach:}

\section{IIA. Column studies:}

Two soils (Quakertown and Adelphia) which had been the subject of previous studies in Professor Rixey's lab were chosen for these initial investigations. Quakertown is a silty loam with an organic matter content of $3.9 \%$. Adelphia is a sandy loam soil with a organic content 
of $1.4 \%$. The columns were prepared by exposing the soils to the test organics in a constantly flowing water stream in order to mimic environmental exposure. In short, water which contained benzene $(\sim 120 \mathrm{ppm})$, toluene $(45 \mathrm{ppm}), m$-xylene $(40 \mathrm{ppm})$, naphthalene $(8 \mathrm{ppm})$, fluorene $(0.6 \mathrm{ppm})$ and phenanthrene $(0.4 \mathrm{ppm})$ was flowed through a column packed with $\sim 50$ grams of the test soil at a linear rate of $\sim 120 \mathrm{~cm} /$ day. Exposure time was either 2 weeks or 8 months. At the end of this period of exposure time, one set of the columns was then desorbed with clean water for an additional six weeks to elute the organics which had not become bound to the soil matrices. Samples from the top and bottom ends of each column were then shipped by overnight air from Prof. Rixey's lab to Dr. Hawthorne's lab, and were extracted by the selective SFE method described below. A duplicate set of columns was also prepared and handled in an identical manner except that no water desorption step of the organics was performed.

\section{II.B. MGP (PAH-contaminated) Soils.}

Soil samples from a historically-contaminated MGP site were provided by the Gas Research Institute (GRI). The soils were collected (and homogenized) before remediation was begun, and after 147 days of active bioremediation. Each sample was subjected to selective SFE analyses using the selective conditions described below.

\section{II.C. Selective SFE Conditions.}

A major effort in these studies was to develop SFE conditions which could selectively extract pollutant organics which ranged from very weakly-bound to very tightly-bound to the soil matrix. Both the pressure of the carbon dioxide used for SFE and the temperature of the extraction affects the solubility and extraction rates. To determine appropriate extraction conditions, initial extractions at several different SFE conditions were performed both on the column samples from Prof. Rixey's lab, and on the soils from the MGP (PAH-contaminated) remediation site. Based on these initial experiments, the SFE conditions listed in Tables I and II were selected. To verify that all of the BTEX and PAHs present on the soil had been extracted by the SFE conditions listed in Table I, the residue after SFE for each column soil sample was also extracted for 18 hours with pure methylene chloride. For the MGP site samples, the conditions in Table II were verified to yield complete extraction of the contaminant PAHs by performing additional extractions with methylene chloride modified $\mathrm{CO}_{2}$, and finally by extracting the SFE residue with methylene chloride for 18 hours.

For both the column samples and the MGP soils, the extracts of the soil residues showed no significant (non-detectable) levels of the contaminant organics after the SFE procedures listed in Tables I and II, respectively. Therefore, the subsequent discussions will focus on the SFE-extracted organics.

\section{IID. Analytical Parameters.}

SFE of the column soils was performed using an ISCO SFX-210 instrument with a coaxially-heated outlet restrictor set at $80^{\circ} \mathrm{C}$ which controlled the flow rate at $\sim 1.2 \mathrm{~mL} / \mathrm{min}$ (measured at the pump). Extracted analytes were collected in $\sim 15 \mathrm{~mL}$ of methylene chloride, which was previously shown to yield quantitative collection of the test species used. For the column studies, the collection solvent was spiked with deuterated analogs of each test species to serve as quantitative internal standards. Every sample used in this study was extracted in 
triplicate with separate extracts collected for each different SFE condition described in Table I. Immediately after extraction, the extracts were analyzed by GC/MS (Hewlett-Packard Model 5973) with selected ion monitoring for the molecular ion of each test compound and deuterated internal standard. Quantitation was based on calibration curves constructed from standard solutions analyzed in the same manner.

The procedure above yielded the mass of each analyte in each selective SFE fraction. Additional extractions were performed in an identical manner on the column samples during which fractions were collected at $5,10,20,40$, and 60 minutes of each selective SFE fraction. These fractions were analyzed in the same manner to yield the shape of the extraction curves (discussed in more detail below).

For the MGP site soil, SFE extractions were performed as outlined in Table II using the same SFE instrument except that an outlet restrictor which allowed $\sim 1.5 \mathrm{~mL} / \mathrm{min}$ of extractant flow rate was used. Extracts were analyzed by GC with flame ionization detection (FID) after the addition of $n$-undecane and $n$-tricosane as internal standards. Samples were extracted and analyzed before the site was opened (day 0 ) and after 147 days of bioremediation.

\section{Results:}

\section{IIIA. Column Studies.}

Representative results (in $\mu \mathrm{g}$ of test analyte per $\mathrm{g}$ of soil on a dry weight basis) for both soils after exposure for 2 weeks and 8 months, and with and without 6 weeks of desorption with pure water after the exposure step are given in Tables III to VIII along with results from sub-samples taken from the inlet and outlet ends of the soil columns. In addition, the extraction rate data (as \% extracted vs. time) are given in Figures 1-8. Since the goal of these initial investigations was to determine the potential usefulness of selective SFE to mimic soil/water partitioning behavior of organic pollutants, and since additional studies (being performed under separate funding) will involve more intensive data evaluation and interpretation, only the major features of the results will be discussed here.

Tables III and IV show the extraction results for both soils after 8 months of exposure to the contaminated water, but with no subsequent desorption with clean water. The tables also compare the results obtained from sub-samples taken from the inlet and outlet ends of the columns. Note that the majority of each species is found in the weakly-bound fraction for both soils, both at the inlet and outlet ends. Comparing the actual concentrations found at the inlet and outlet ends of the columns for benzene, toluene, and $m$-xylene is difficult because of the high variability of the quantitative data which results because the weakly-bound solutes are lost to evaporation from the sample between the triplicate experiments performed on each sample. (Note that this is not a problem for samples that have undergone the 6-week desorption step as presented in subsequent tables.) However, the less volatile species do not experience these losses, and the concentrations found in the inlet and outlet ends of the Adelphia soil were virtually identical for naphthalene, fluorene, and phenanthrene. For Quakertown soil (Table IV), the concentrations of all of the test species except phenanthrene agree quite well for the inlet and outlet ends of the columns, however the phenanthrene concentration is substantially lower at the column outlet, suggesting that phenanthrene is somewhat retained on the soil at the column inlet during the column preparation. Similar results for the outlet and inlet ends of the columns were found for subsequent samples (Tables V to VIII). Since the inlet end of the 
columns will be closest to equilibrium during the sorption and desorption steps, subsequent discussions will deal only with samples taken from the inlet ends.

The distribution of the various pollutants among the weak to very strong sites for the 8 month (not desorbed) samples is most easily seen in Figures 1 to 4. For both the Adelphia and Quakertown samples (and for both the inlet and outlet of both samples), the largest fraction of all of the test organics were found in the weakly-bound fraction ( 0 to 60 minutes in Figures 1 to 4). Only a few percent of the molecules were found in the moderate-bound fraction (60 to 120 minutes), and very low percentages were found in the more tightly-bound fractions.

Very different behavior is seen for the extraction of replicate columns that were desorbed with pure water for 6 weeks after the 8-month exposure period (Tables V and VI, and Figures 5 and 6). After replicate samples had undergone the 6-week desorption with pure water, the percent of each organic found in the more strongly-bound fractions (moderately-bound at 60120 minutes, strongly-bound at 120-180 minutes, and very strongly-bound at 180-210 minutes) increased dramatically for both samples as shown in Figures 5 and 6 . (Note that the figures are on a percent, not mass basis.) These results based on selective SFE are exactly as expected based on column water leaching studies, and provide the first evidence that selective SFE is a viable approach to determining soil/water interactions that occur over long periods of time.

Depending on whether or not the soil was desorbed with pure water after the 8-month exposure period, the mass of each analyte found on the soils is substantially different. As shown in Table V for the Adelphia soil and Table VI for the Quakertown soil, the desorbed soil showed only very low concentrations of each organic (except phenanthrene) in the weaklybound fraction. In contrast, the same soil that had not undergone desorption showed nearly all of the molecules in the weak fraction. The effect of the 6-week desorption period is more clearly seen in Table IX. First, a comparison of the total mass of each analyte shows that only $\sim 2$ to $10 \%$ of the organics (except for $\sim 40 \%$ for phenanthrene) that were originally found on the soils after 8 months of exposure remain on the soil after the subsequent 6-week desorption with pure water. Most importantly, the analytes that are removed by the water desorption step are those that extract most rapidly under the mildest SFE conditions. As shown in Table IX, the non-desorbed samples have much higher quantities of all of the analytes in the weakly bound fractions than the desorbed soils, while the concentrations in the moderately-bound fractions are more similar. Finally, the mass of each pollutant found in the strong and very strong sites are very similar (likely within analytical error), regardless of whether the soil was subjected to the 6-week desorption period or not.

Additional evidence that selective SFE mimics soil/water sorption/desorption behavior is found by comparing the extraction rates of the higher and lower molecular weight species. For the non-desorbed samples (Figures 1 and 2), the higher molecular weight organics are more difficult to extract as would be expected based on their lower solubility in supercritical $\mathrm{CO}_{2}$. However, for the soils that were desorbed for 6 weeks, the lower molecular weight organics extract more slowly than the higher molecular weight species (in direct contrast to their behavior expected based on solubility in $\mathrm{CO}_{2}$ ). These observations are exactly what would be expected if the pore diffusion model of solute binding to soils is correct--i.e., the lower molecular weight organics that remain bound to the soil after leaching with pure water have diffused further into the matrix than the higher molecular weight species, and thus show slower extraction under the SFE conditions.

These results clearly demonstrate that the selective SFE is mimicking the desorption 
processes that actually occur in the environment for contaminated soils that are leached by ground water.

The results of the soils that were exposed for two weeks followed by 6 weeks of desorption are shown in Tables VII and VIII, and Figures 7 and 8. (Note that fluorene and phenanthrene were not included in these columns.) As would be expected based on the lower exposure time, the quantities of each pollutant are $\sim 1 / 2$ as much as for the samples that had been exposed for 8 months (again, followed by 6 weeks of desorption). However, a surprising result is that the percentage of each pollutant found in each fraction (Figures 7 and 8) are similar to those found in the 8 month exposed samples (Figures 5 and 6). This result may be an artifact of the analysis, since the mass of analytes being determined is very near the detection limits. In addition, the presence of other isomers of $\mathrm{C}_{2}$-benzenes (besides the test species $m$-xylene) in proportions found in gasoline indicate that the two "clean" soils actually have very low levels of hydrocarbon contamination that is found in the tightly-bound sites. Additional studies will be performed (under future funding) to investigate these possibilities. Based on initial determinations, it is expected that the organics found in the tighter sites for the 2-week exposure samples are also present in the "blank" soils, and thus the major fraction of the organics found in the 2-week samples from the laboratory exposures are found only in the weaker sites. In any case, the results discussed above for the samples exposed for 8 months demonstrate that selective SFE behavior parallels the long-term behavior found in contaminated soil/water systems.

\section{III.B. MGP (PAH-contaminated) Soils.}

Initial comparisons between our quantitative results based on the SFE protocol listed in Table II, and conventional (Soxhlet extraction) methods were performed to validate the analytical methods. As shown in Table $\mathrm{X}$, the agreement between samples analyzed by conventional methods and the step-wise SFE approach was very good.

The day 0 (untreated) sample was then extracted using the selective SFE conditions to determine the fractions of each PAH found in weak to very tight sites. As shown in Table XI, the lower molecular weight PAHs are found 90\% in the weak binding sites, with the remaining molecules distributed over the three tighter fractions. As the molecular weight of the PAH increases, the major fraction shifts from the weak sites to the moderate sites, with lesser amounts found in the strong and very strong sites.

The distribution of the same PAHs on the soil after 147 days of bioremediation was determined using identical SFE conditions. As shown in Table XII and Figure 9, the majority of PAHs that were removed by the bioremediation correspond to the molecules found on the weaker sites by selective SFE. For example, the concentration of naphthalene found in the weak sites from the untreated sample decreased from 85 to $13 \mathrm{ppm}$, while the concentrations of the naphthalene found on the strong and very strong sites was not changed after 147 days of bioremediation. Similar results were seen for the other PAHs on the soil (Table XII and Figure 9).

When the total PAH concentration is determined in each SFE fraction before and after remediation, the overall removal of the PAHs from weak sites is $\sim 80 \%$, removal from moderate sites is $\sim 50 \%$, and there is no removal of the PAHs found on the strong and very 
strong sites, as is clearly shown in Figure 9 and Table XII.

Finally, selective SFE behavior of the PAHs from the untreated soil was used to estimate the fraction of PAHs which would be removed during the 147 day bioremediation process. This was accomplished by (somewhat arbitrarily) assuming that the weakly-bound PAHs (as determined by their presence in the first SFE fraction) would be removed by bioremediation, but the more strongly-bound PAHs would not be removed. The results of the PAH concentrations predicted to remain after bioremediation (i.e., those in the three stronger SFE fractions) and the actual concentrations found to remain in the soil after bioremediation are shown in Table XIII. Overall, the predictions made by SFE are quite good for individual PAHs. However, when the amount of removal predicted by SFE is based on total PAHs, the predicted PAH concentrations remaining on the soil is virtually identical to the actual value found in the bioremediated soil.

Clearly, the results in Table XIII demonstrate a high potential for selective SFE to provide a rapid laboratory test to predict and investigate the effects of remediation processes. Future studies (under separate funding) are anticipated to use selective SFE to monitor and predict bioremediation behavior of $\sim$ four additional MGP sites located throughout the United States. 
Table I: Selective SFE Conditions for Column Sorption/Desorption Samples

\begin{tabular}{lcccc}
\hline binding & time (min) & pressure (bar) & temp. $\left({ }^{\circ} \mathrm{C}\right)$ & extraction fluid \\
weak & $0-60$ & 400 & 50 & $\mathrm{CO}_{2}$ \\
medium & $60-120$ & 400 & 100 & $\mathrm{CO}_{2}$ \\
strong & $120-180$ & 400 & 150 & $\mathrm{CO}_{2}$ \\
very strong & $180-210$ & 400 & 150 & $\mathrm{CO}_{2} / \mathrm{CH}_{2} \mathrm{Cl}_{2}$ \\
& & & & \\
\hline
\end{tabular}

Table II: Selective SFE Conditions for Manufactured Gas Plant Soils

\begin{tabular}{lcccc}
\hline binding & time (min) & pressure (bar) & temp. $\left({ }^{\circ} \mathrm{C}\right)$ & extraction fluid \\
weak & $0-30$ & 120 & 50 & $\mathrm{CO}_{2}$ \\
medium & $30-60$ & 400 & 50 & $\mathrm{CO}_{2}$ \\
strong & $60-90$ & 400 & 100 & $\mathrm{CO}_{2}$ \\
very strong & $90-120$ & 400 & 150 & $\mathrm{CO}_{2}$ \\
& & & & \\
\hline
\end{tabular}


Table III: Concentration $(\mu \mathrm{g} / \mathrm{g}) \pm$ SD of Organics in Each SFE Fraction from Adelphia Soil (8 month sorption, no desorption)

\begin{tabular}{|c|c|c|c|c|c|}
\hline \multicolumn{6}{|l|}{ column inlet } \\
\hline & weak & medium & strong & very strong & total \\
\hline benzene & $10.6 \pm 7.1$ & $1.1 \pm 0.3$ & $0.34 \pm 0.19$ & $0.03 \pm 0.03$ & $12.1 \pm 7.2$ \\
\hline toluene & $7.2 \pm 4.1$ & $0.6 \pm 0.5$ & $0.44 \pm 0.47$ & $0.10 \pm 0.07$ & $8.4 \pm 5.3$ \\
\hline$m$-xylene & $21.7 \pm 6.3$ & $0.94 \pm 0.14$ & $0.20 \pm 0.14$ & $0.13 \pm 0.02$ & $23.0 \pm 6.9$ \\
\hline fluorene & $16.0 \pm 3.6$ & $1.05 \pm 0.02$ & $0.04 \pm 0.02$ & $0.02 \pm 0.01$ & $17.1 \pm 4.0$ \\
\hline phenanthrene & $35.8 \pm 6.6$ & $4.5 \pm 0.1$ & $0.2 \pm 0.1$ & $0.10 \pm 0.03$ & $40.7 \pm 8.3$ \\
\hline \multicolumn{6}{|l|}{ column outlet } \\
\hline & weak & medium & strong & very strong & total \\
\hline benzene & $2.8 \pm 3.4$ & $0.8 \pm 0.1$ & $0.3 \pm 0.2$ & $0.01 \pm 0.01$ & $4.0 \pm 3.5$ \\
\hline toluene & $2.2 \pm 2.7$ & $0.24 \pm 0.03$ & $0.14 \pm 0.03$ & $0.1 \pm 0.1$ & $2.7 \pm 2.8$ \\
\hline$m$-xylene & $9.8 \pm 7.6$ & $0.7 \pm 0.1$ & $0.2 \pm 0.1$ & $0.10 \pm 0.03$ & $10.7 \pm 7.7$ \\
\hline fluorene & $16.2 \pm 2.8$ & $1.5 \pm 0.1$ & $0.1 \pm 0.1$ & $0.02 \pm 0.01$ & $17.8 \pm 2.2$ \\
\hline phenanthrene & $35.3 \pm 3.5$ & $5.5 \pm 0.1$ & $0.27 \pm 0.14$ & $0.13 \pm 0.05$ & $41.3 \pm 1.2$ \\
\hline
\end{tabular}


Table IV: Concentration $(\mu \mathrm{g} / \mathrm{g}) \pm \mathrm{SD}^{\mathrm{a}}$ of Organics in Each SFE Fraction from Quakertown Soil (8 month sorption, no desorption)

column inlet

weak

medium

strong

very strong

total

$\begin{array}{llllll}\text { benzene } & 14.7 \pm 9.97 & 3.6 \pm 1.3 & 0.79 \pm 0.81 & 0.03 \pm 0.01 & 19.1 \pm 9.3 \\ \text { toluene } & 13.0 \pm 7.2 & 4.2 \pm 0.4 & 0.42 \pm 0.42 & 0.13 \pm 0.15 & 17.8 \pm 7.1 \\ m \text {-xylene } & 37.3 \pm 13.0 & 17.4 \pm 0.3 & 0.46 \pm 0.32 & 0.26 \pm 0.11 & 55.4 \pm 12.5 \\ \text { fluorene } & 22.6 \pm 7.4 & 18.6 \pm 0.03 & 0.13 \pm 0.03 & 0.07 \pm 0.02 & 41.4 \pm 2.5 \\ \text { phenanthrene } & 45.7 \pm 14.6 & 50.4 \pm 0.1 & 0.51 \pm 0.05 & 0.31 \pm 0.06 & 97.0 \pm 2.4\end{array}$

$\underline{\text { column outlet }}$

weak

medium

strong

very strong

total

benzene

$18.1 \pm 10.8$

$8.3 \pm 4.6$

$0.46 \pm 0.20$

$0.04 \pm 0.00$

$0.09 \pm 0.03$

$26.9 \pm 15.5$

$13.2 \pm 5.3$

$10.15 \pm 0.03$

$0.29 \pm 0.03$

$0.19 \pm 0.02$

$23.8 \pm 10.1$

$m$-xylene

$29.4 \pm 5.6$

$0.44 \pm 0.14$

$0.07 \pm 0.04$

$67.3 \pm 16.5$

fluorene

$26.61 \pm 0.02$

$0.14 \pm 0.02$

$0.23 \pm 0.13$

$36.3 \pm 1.9$

$12.5 \pm 0.9$

$44.99 \pm 0.06$

$0.38 \pm 0.06$

$58.3 \pm 4.7$ 
Table V: Concentration $(\mu \mathrm{g} / \mathrm{g}) \pm \mathrm{SD}^{\mathrm{a}}$ of Organics in Each SFE Fraction from Adelphia Soil (8 month sorption, 6 weeks desorption)

\begin{tabular}{|c|c|c|c|c|c|}
\hline & weak & medium & strong & very strong & total \\
\hline benzene & $0.26 \pm 0.08$ & $0.61 \pm 0.05$ & $0.32 \pm 0.05$ & $0.05 \pm 0.01$ & $1.23 \pm 0.19$ \\
\hline toluene & $0.09 \pm 0.01$ & $0.16 \pm 0.01$ & $0.10 \pm 0.01$ & $0.03 \pm 0.00$ & $0.38 \pm 0.02$ \\
\hline$m$-xylene & $0.12 \pm 0.02$ & $0.21 \pm 0.01$ & $0.07 \pm 0.01$ & $0.06 \pm 0.02$ & $0.46 \pm 0.03$ \\
\hline fluorene & $1.14 \pm 0.55$ & $0.41 \pm 0.01$ & $0.02 \pm 0.01$ & $0.00 \pm 0.00$ & $1.57 \pm 0.58$ \\
\hline phenanthrene & $10.2 \pm 2.4$ & $4.65 \pm 0.07$ & $0.19 \pm 0.07$ & $0.07 \pm 0.05$ & $15.1 \pm 2.8$ \\
\hline
\end{tabular}

${ }^{\text {aStandard deviation }}$ 
Table VI: Concentration $(\mu \mathrm{g} / \mathrm{g}) \pm \mathrm{SD}^{\mathrm{a}}$ of Organics in Each SFE Fraction from Quakertown Soil (8 month sorption, 6 week desorption)

\begin{tabular}{lccccc}
\hline & & & & & \\
& weak & medium & strong & very strong & \\
benzene & $0.26 \pm 0.08$ & $0.70 \pm 0.04$ & $0.40 \pm 0.08$ & $0.07 \pm 0.01$ & $1.43 \pm 0.10$ \\
toluene & $0.08 \pm 0.04$ & $0.29 \pm 0.03$ & $0.17 \pm 0.03$ & $0.03 \pm 0.01$ & $0.59 \pm 0.03$ \\
$\begin{array}{l}m \text {-xylene } \\
\text { fluorene }\end{array}$ & $0.22 \pm 0.11$ & $0.63 \pm 0.09$ & $0.22 \pm 0.09$ & $0.05 \pm 0.01$ & $1.13 \pm 0.09$ \\
phenanthrene & $3.75 \pm 1.95$ & $4.71 \pm 0.13$ & $0.15 \pm 0.13$ & $0.05 \pm 0.06$ & $8.67 \pm 1.34$ \\
& $17.3 \pm 10.6$ & $27.1 \pm 0.3$ & $0.57 \pm 0.26$ & $0.16 \pm 0.11$ & $45.3 \pm 8.8$ \\
\hline
\end{tabular}

${ }^{\text {aStandard deviation }}$ 
Table VII: Concentration $(\mu \mathrm{g} / \mathrm{g}) \pm$ SD $^{\mathrm{a}}$ of Organics in Each SFE Fraction from Adelphia Soil (2 week sorption, 6 weeks desorption)

\begin{tabular}{lccccc}
\hline & & & & & \\
& weak & medium & strong & very strong & \\
benzene & & & & & \\
toluene & $0.24 \pm 0.09$ & $0.31 \pm 0.12$ & $0.24 \pm 0.09$ & $0.03 \pm 0.02$ & $0.83 \pm 0.31$ \\
$m$-xylene & $0.04 \pm 0.01$ & $0.07 \pm 0.01$ & $0.04 \pm 0.01$ & $0.02 \pm 0.01$ & $0.17 \pm 0.04$ \\
naphthalene & $0.06 \pm 0.00$ & $0.08 \pm 0.01$ & $0.03 \pm 0.01$ & $0.04 \pm 0.02$ & $0.21 \pm 0.05$ \\
& $0.14 \pm 0.02$ & $0.12 \pm 0.01$ & $0.03 \pm 0.01$ & $0.00 \pm 0.00$ & $0.30 \pm 0.06$ \\
\hline
\end{tabular}

${ }^{\text {aStandard deviation }}$ 
Table VIII: Concentration $(\mu \mathrm{g} / \mathrm{g}) \pm \mathrm{SD}^{\mathrm{a}}$ of Organics in Each SFE Fraction from Quakertown Soil (8 month sorption, 6 week desorption)

\begin{tabular}{lccccc}
\hline & & & & & \\
& weak & medium & strong & very strong & total \\
benzene & & & & & \\
toluene & $0.31 \pm 0.09$ & $0.45 \pm 0.12$ & $0.26 \pm 0.11$ & $0.05 \pm 0.01$ & $1.07 \pm 0.20$ \\
$m$-xylene & $0.04 \pm 0.02$ & $0.13 \pm 0.02$ & $0.02 \pm 0.02$ & $0.02 \pm 0.01$ & $0.20 \pm 0.08$ \\
naphthalene & $0.05 \pm 0.02$ & $0.28 \pm 0.01$ & $0.03 \pm 0.01$ & $0.02 \pm 0.01$ & $0.38 \pm 0.08$ \\
\hline
\end{tabular}

aStandard deviation 
Table IX: Comparison of the Concentration $(\mu \mathrm{g} / \mathrm{g})$ of Each Organic in the Selective SFE Fractions on Soils Exposed for 8 Months, With and Without 6 Weeks of Desorption.

Adelphia

\begin{tabular}{|c|c|c|c|c|c|c|c|c|c|c|}
\hline \multirow[b]{2}{*}{ desorbed? } & \multicolumn{2}{|c|}{ weak } & \multicolumn{2}{|c|}{ medium } & \multicolumn{2}{|c|}{ strong } & \multicolumn{2}{|c|}{ very strong } & \multirow[t]{2}{*}{ total $\mu \mathrm{g} / \mathrm{g}+\mathrm{SD}^{\mathrm{a}}$} & \multirow[t]{2}{*}{ total $\mu \mathrm{g} / \mathrm{g}+\mathrm{SD}$} \\
\hline & yes & no & yes & no & yes & no & yes & no & & \\
\hline benzene & 0.26 & 10.57 & 0.61 & 1.13 & 0.32 & 0.34 & 0.05 & 0.03 & $1.23 \pm 0.19$ & $12.09 \pm 7.24$ \\
\hline toluene & 0.09 & 7.16 & 0.16 & 0.62 & 0.10 & 0.44 & 0.03 & 0.10 & $0.38 \pm 0.02$ & $8.36 \pm 5.25$ \\
\hline$m$-xylene & 0.12 & 21.66 & 0.21 & 0.94 & 0.07 & 0.20 & 0.06 & 0.13 & $0.46 \pm 0.03$ & $22.96 \pm 6.87$ \\
\hline naphthalene & 0.27 & 30.44 & 0.26 & 1.14 & 0.08 & 0.13 & 0.01 & 0.04 & $0.63 \pm 0.06$ & $31.76 \pm 4.55$ \\
\hline fluorene & 1.14 & 16.96 & 0.41 & 1.05 & 0.02 & 0.04 & 0.00 & 0.02 & $1.57 \pm 0.58$ & $17.08 \pm 3.95$ \\
\hline phenanthrene & 10.2 & 35.79 & 4.65 & 4.49 & 0.19 & 0.21 & 0.07 & 0.10 & $15.09 \pm 2.77$ & $40.65 \pm 8.32$ \\
\hline \multicolumn{11}{|l|}{ Quakertown } \\
\hline & \multicolumn{2}{|c|}{ weak } & \multicolumn{2}{|c|}{ medium } & \multicolumn{2}{|c|}{ strong } & \multicolumn{2}{|c|}{ very strong } & \multirow[t]{2}{*}{ total $\mu \mathrm{g} / \mathrm{g} \pm \mathrm{SD}$} & \multirow[t]{2}{*}{$\underline{\text { total } \mu \mathrm{g} / \mathrm{g} \pm \mathrm{SD}}$} \\
\hline desorbed? & yes & no & yes & no & yes & no & yes & no & & \\
\hline benzene & 0.26 & 14.72 & 0.70 & 3.58 & 0.40 & 0.79 & 0.07 & 0.03 & $1.43 \pm 0.10$ & 19.13 \\
\hline toluene & 0.08 & 13.04 & 0.29 & 4.15 & 0.17 & 0.42 & 0.03 & 0.13 & $0.59 \pm 0.03$ & $17.78 \pm 7.05$ \\
\hline$m$-xylene & 0.22 & 37.26 & 0.63 & 17.41 & 0.22 & 0.46 & 0.05 & 0.26 & $1.13 \pm 0.09$ & \pm 12.54 \\
\hline naphthalene & 0.79 & 44.66 & 1.25 & 24.32 & 0.38 & 0.58 & 0.09 & 0.24 & $2.54 \pm 0.41$ & \pm 2.00 \\
\hline fluorene & 3.75 & 22.63 & 4.71 & 18.57 & 0.15 & 0.13 & 0.05 & 0.07 & $8.67 \pm 1.34$ & 41.42 \\
\hline phenanthrene & 17.3 & 45.65 & 27.14 & 50.36 & 0.57 & 0.51 & 0.16 & 0.31 & $45.25 \pm 8.79$ & $\pm \quad 2.41$ \\
\hline
\end{tabular}

\footnotetext{
${ }^{a}$ Standard deviation
} 
Table X: Day 0 Concentrations (ppm) for MGP Soil: Conventional vs. SFE Analyses

Total SFE Conventional

$\begin{array}{lrr}\text { naphthalene } & 98 & 90 \\ \text { 2-methylnaphthalene } & 162 & 170 \\ \text { fluorene } & 171 & 120 \\ \text { phenanthrene } & 469 & 360 \\ \text { anthracene } & 110 & 110 \\ \text { fluoranthene } & 153 & 120 \\ \text { pyrene } & 254 & 190 \\ \text { benz[a]anthracene } & 89 & 75 \\ \text { chrysene } & 78 & 66 \\ \text { benzo[b,k]pyrene } & 60 & 59 \\ \text { benzo[a]pyrene } & 65 & 50\end{array}$


Table XI: \% of PAHs in Each SFE Fraction for MGP Soil

\begin{tabular}{lcccc} 
& \multicolumn{5}{c}{ \% in each fraction (day 0) } \\
\cline { 2 - 5 } & weak & moderate & strong & very strong \\
naphthalene & 87 & 4.2 & 4.8 & 4.0 \\
2-methylnaphthalene & 93 & 3.8 & 2.2 & 1.4 \\
1-methylnaphthalene & 94 & 3.7 & 0.8 & 0.2 \\
phenanthrene & 72 & 25 & 2.5 & 0.7 \\
anthracene & 65 & 26 & 4.1 & 4.0 \\
fluoranthene & 39 & 53 & 5.9 & 2.0 \\
pyrene & 34 & 57 & 7.2 & 2.0 \\
benz[a]anthracene & 16 & 67 & 13 & 3.3 \\
chrysene & 12 & 68 & 15 & 5.2 \\
benzo $[b, k]$ pyrene & 8.4 & 77 & 11 & 3.1 \\
benzo[a]pyrene & 7.7 & 58 & 25 & 9.0 \\
& 68 & & & \\
Total PAHs & 68 & & 3.9 & 2.6 \\
& & & & \\
\hline
\end{tabular}


Table XII: Distribution of PAHs from MGP Soil in SFE Fractions Before and After Remediation for 147 Days

Concentration (ppm) in each fraction

\begin{tabular}{llll}
\hline weak & moderate & strong & very strong
\end{tabular}

\begin{tabular}{llllll} 
naphthalene & (Day 0) & 85 & 4.1 & 4.7 & 3.9 \\
& (Day 147) & 13 & 1.9 & 4.2 & 3.9 \\
anthracene & (Day 0) & 72 & 29 & 4.5 & 4.3 \\
& (Day 147) & 12 & 9.9 & 3.4 & 4.3 \\
chrysene & (Day 0) & 8.6 & 51 & 11 & 3.8 \\
& (Day 147) & 4.5 & 27 & 11 & 3.5 \\
Total PAHs & (Day 0) & 5740 & 2210 & 331 & 224 \\
& (Day 147) & 1230 & 1020 & 345 & 252 \\
& & & & & \\
\hline
\end{tabular}


Table XIII: Predicted (SFE "Weak" site) and Actual PAH Removal after 147 Days

Concentration (ppm)

\begin{tabular}{lrrr} 
& & \multicolumn{2}{c}{ Remaining after 147 Days } \\
\cline { 2 - 4 } & & Predicted & Actual \\
& Day 0 & 13 & \\
naphthalene & & 12 & 23 \\
2-methylnaphthalene & 97 & 9 & 30 \\
1-methylnaphthalene & 162 & 17 & 25 \\
fluorene & 160 & 133 & 31 \\
phenanthrene & 171 & 38 & 65 \\
anthracene & 469 & 93 & 29 \\
fluoranthene & 110 & 169 & 56 \\
pyrene & 153 & 73 & 107 \\
benz $[a]$ anthracene & 254 & 66 & 50 \\
chrysene & 87 & 54 & 46 \\
benzo[b,k]fluoranthene & 74 & 51 & 39 \\
benzo[a]pyrene & 59 & & 53 \\
Total PAHs & 55 & 2770 & 2850 \\
& 8510 & & \\
\hline
\end{tabular}


Figure Legends: For Figure 9, there is only the original (color) in the folder. 
Figure 1

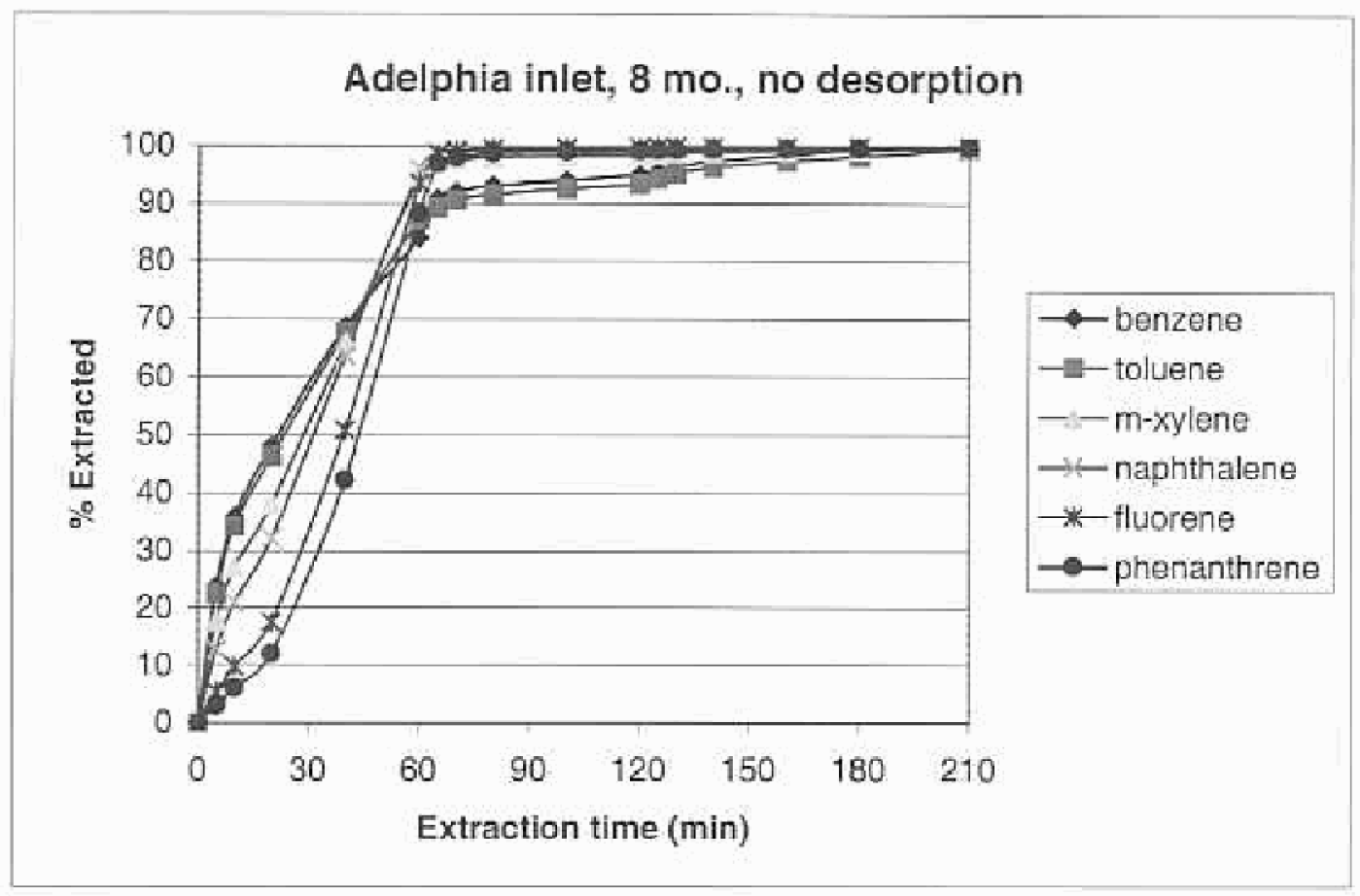


Figure 2

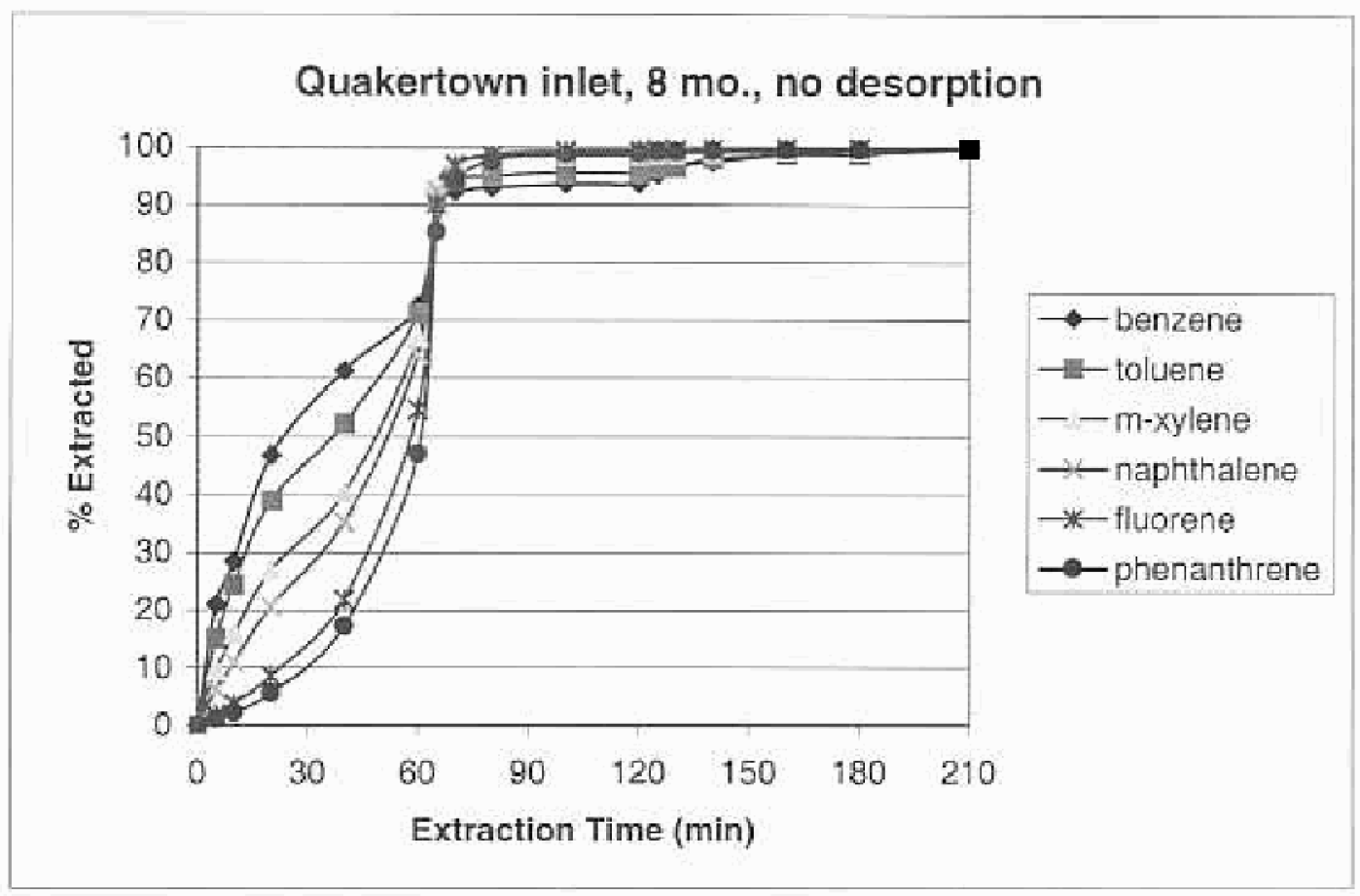


Figure 3

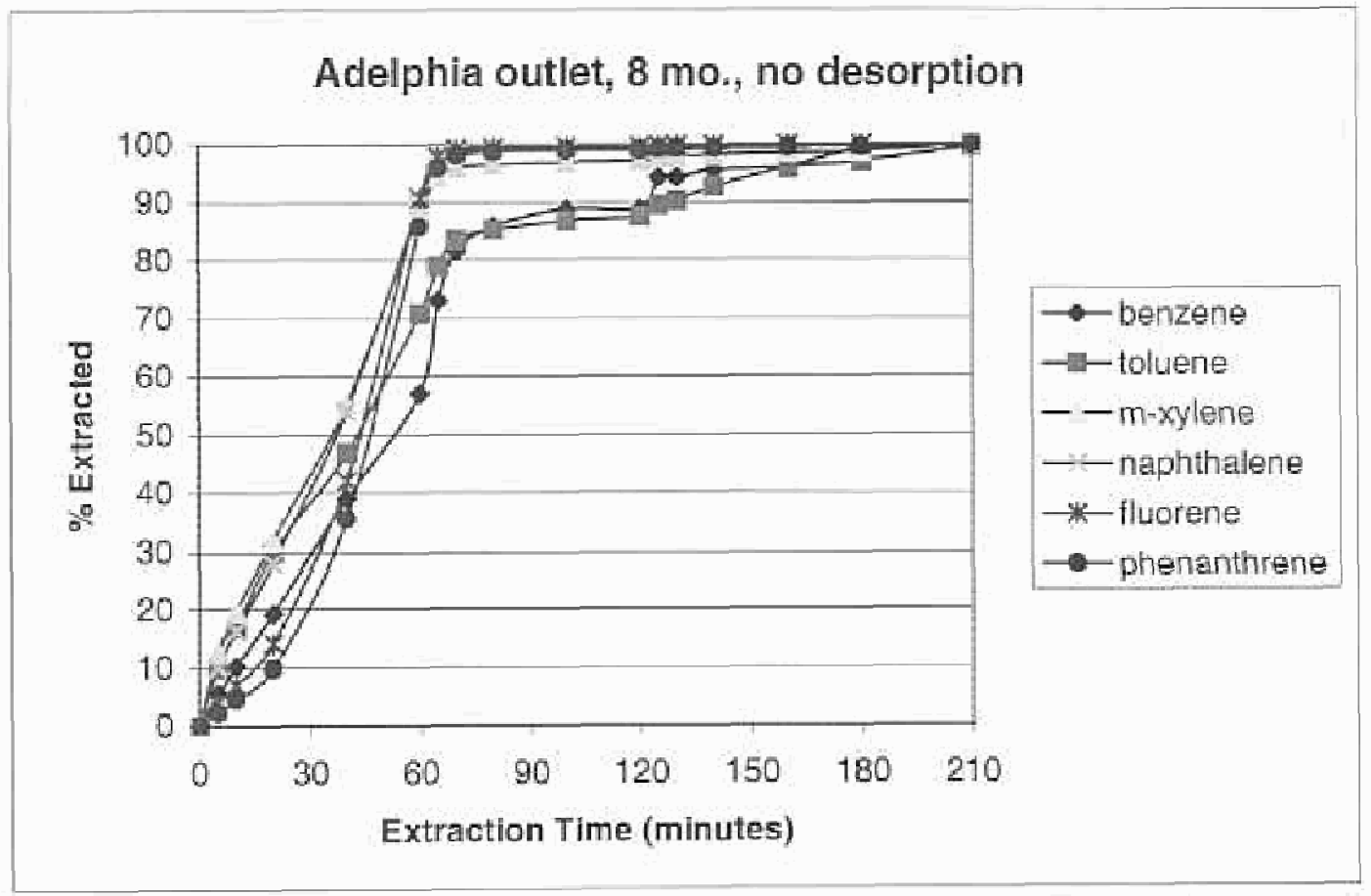


Figure 4

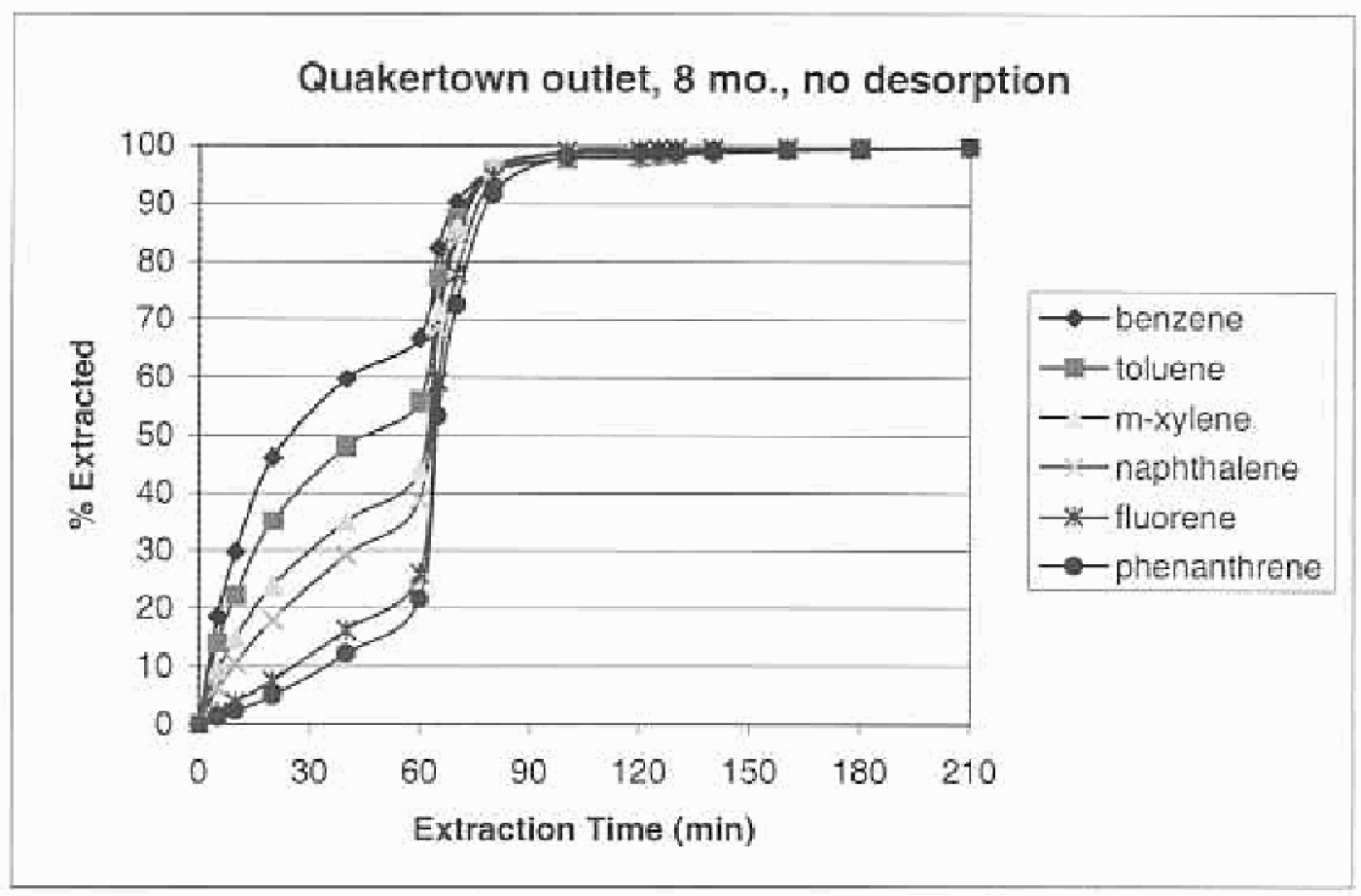


Figure 5

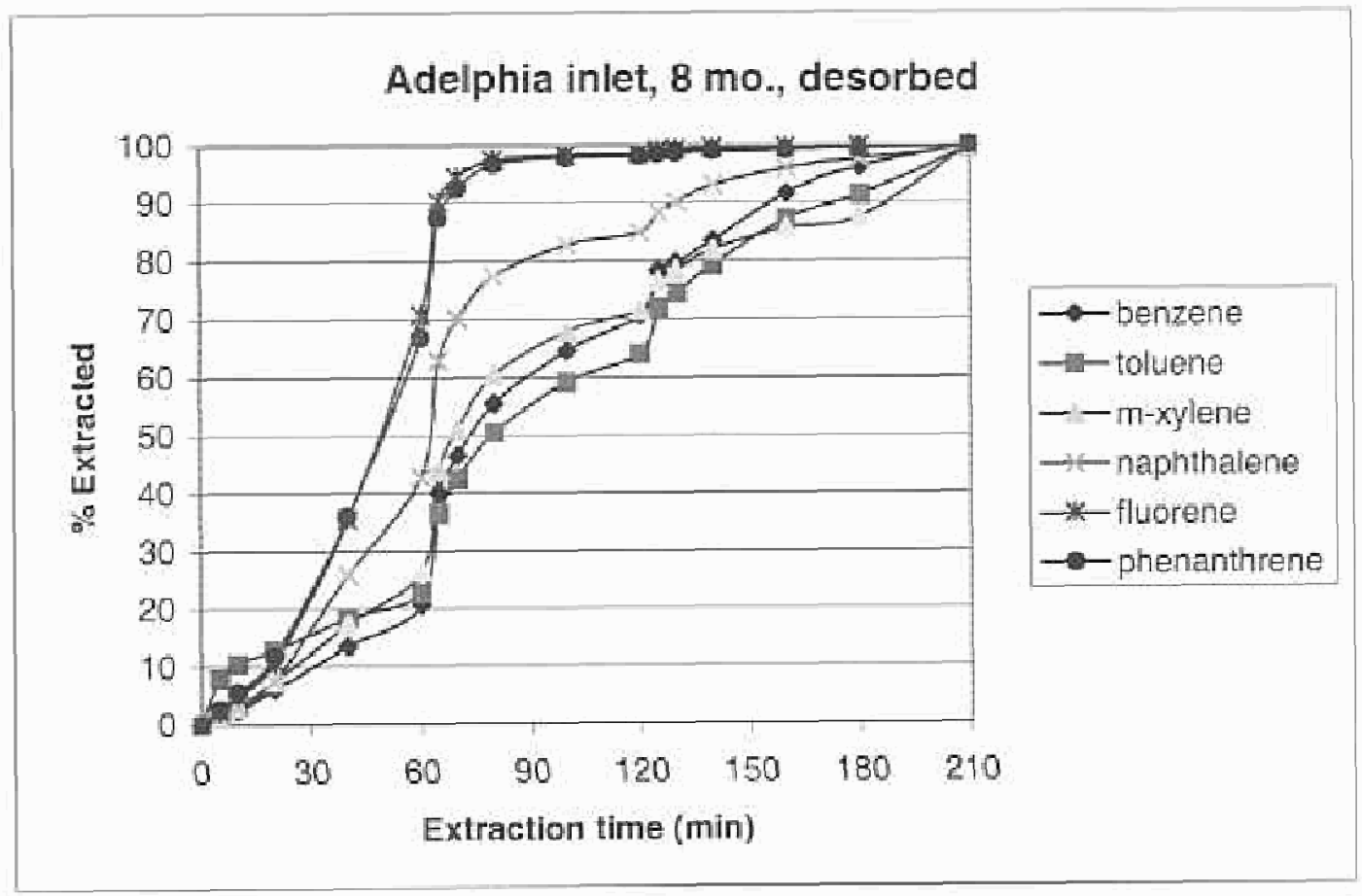


Figure 6

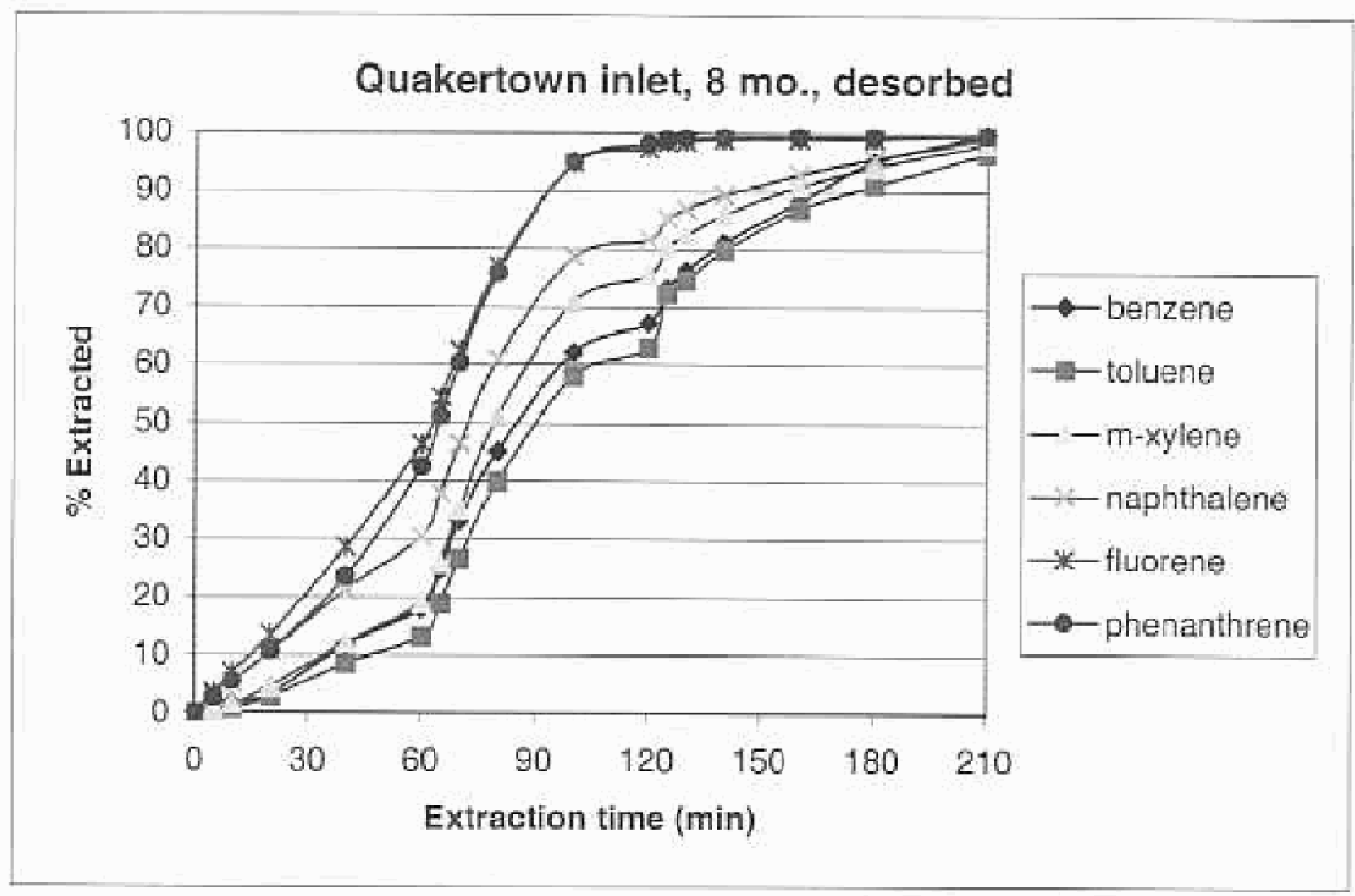


Figure 7

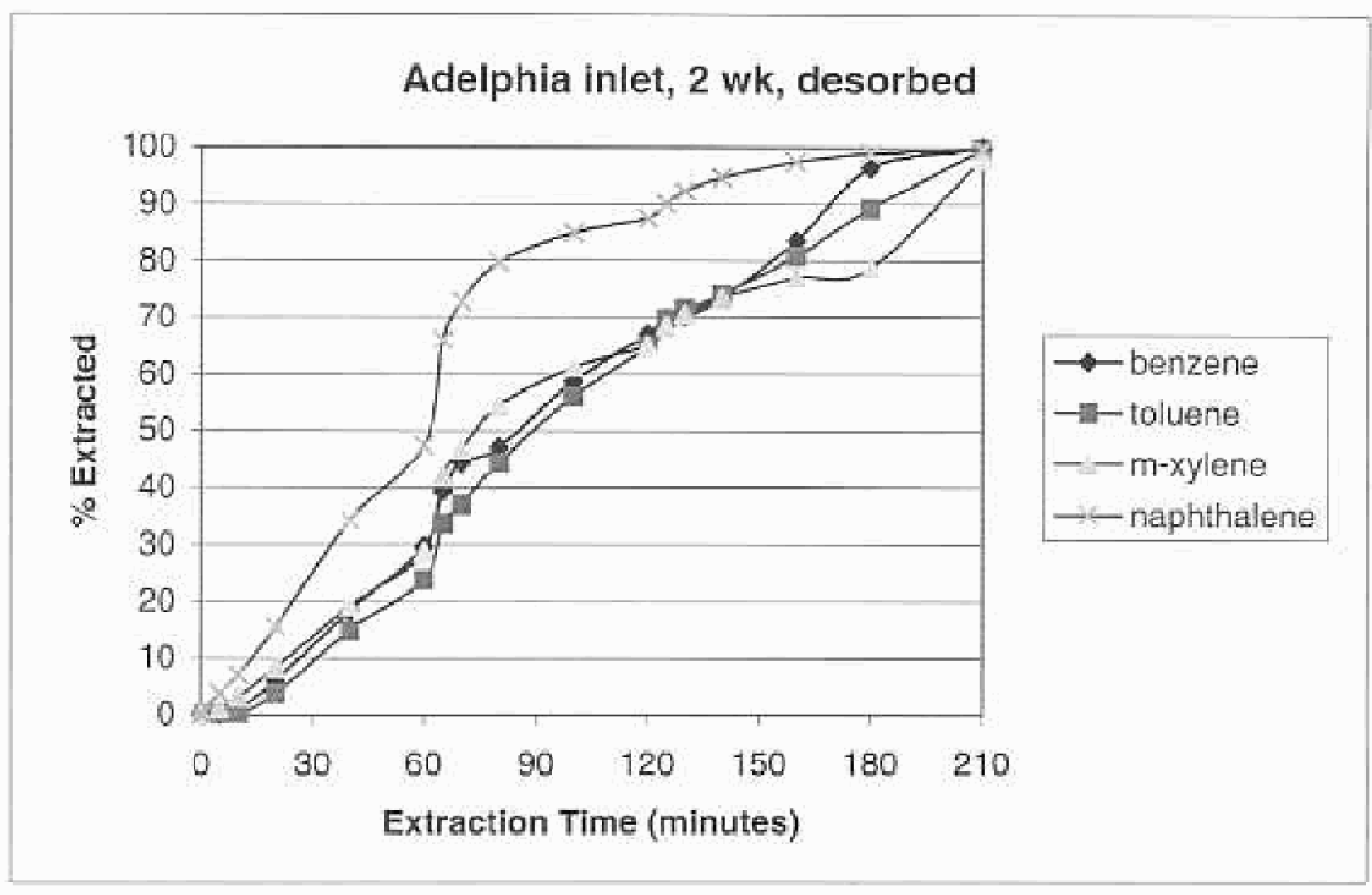


Figure 8

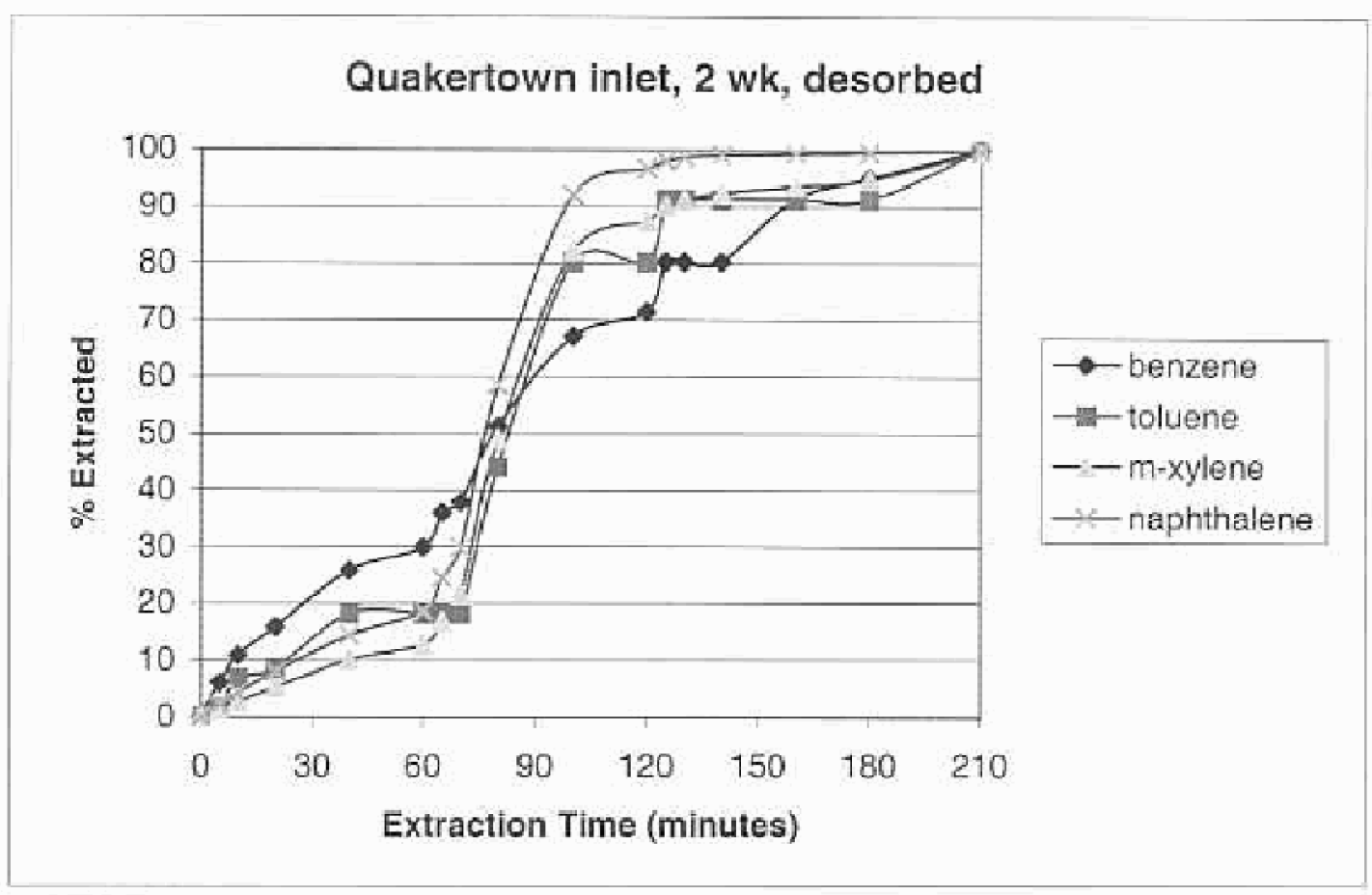


Figure 9

\section{Total PAHs}

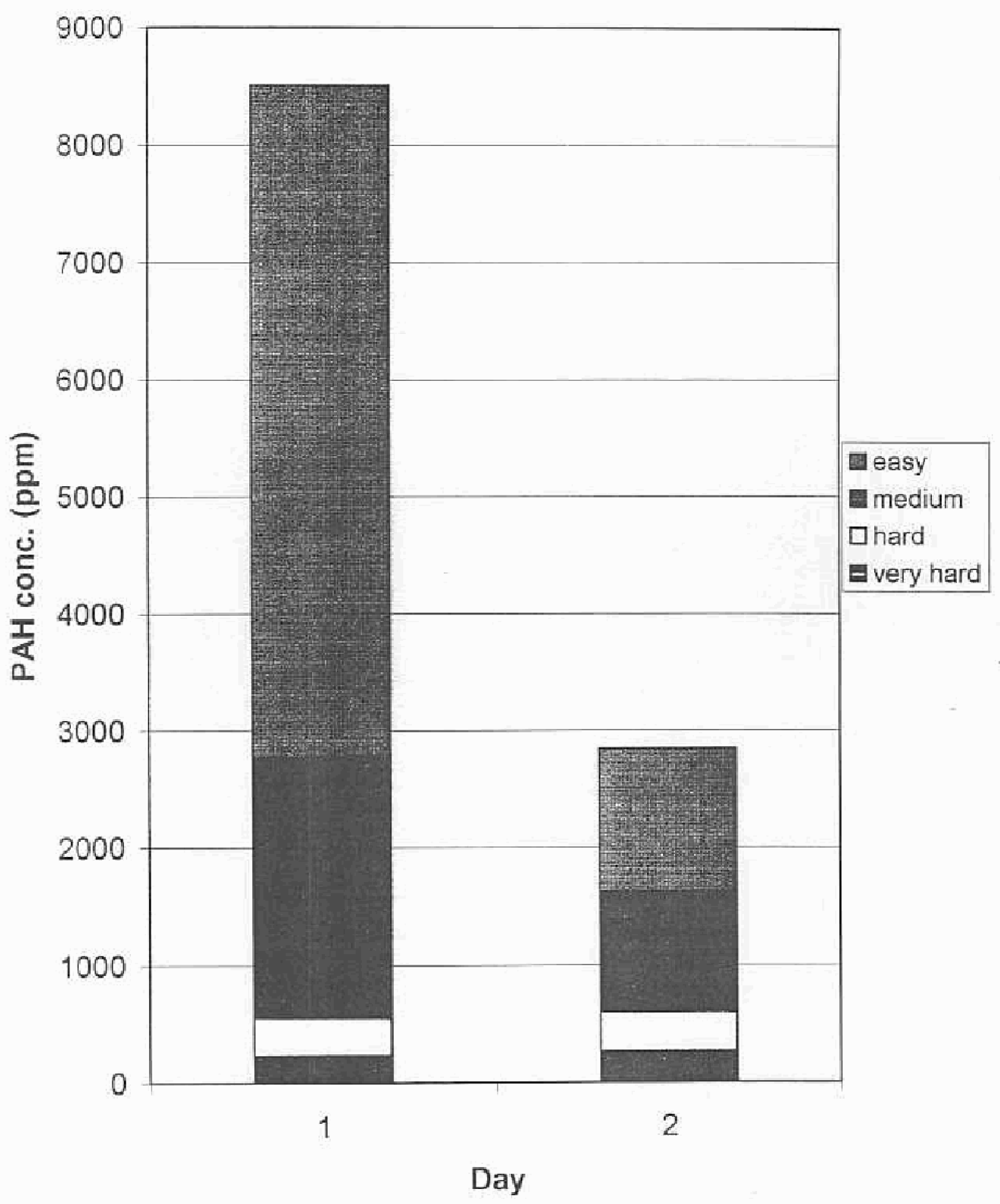

PAH concentrations in four SFE fractions before and after 147 days of remediation 